\title{
Tribology of Titanium-Aluminium Base Nanomultilayer Coatings
}

Totka Bakalova ${ }^{1}$, Nikolay Petkov $^{2}$, Hristo Bahchedzhiev ${ }^{2}$, Petr Louda ${ }^{1}$

${ }^{1}$ Institute for Nanomaterials, Advanced Technologies and Innovation, Technical University of Liberec, Studentská 2, 461 17 Liberec, Czech Republic, E-mail: totka.bakalova@tul.cz

${ }^{2}$ Central Laboratory of Applied Physics, Bulgarian Academy of Sciences, 61, St. Peterburg Blvd. 4000 Plovdiv, Bulgaria. E-mail: petkovnik@gmail.com, hristo_bah@abv.bg

The article presents investigation of the trybological properties, particularly dry friction and friction using a process fluid, of friction pairs of steel ball against titanium-aluminium base nanomultilayer (NML) coatings. The coatings are deposited by cathodic arc evaporation of both pure titanium and aluminium with eighteen percent silicon cathodes. Each multilayer structure consists of 49 bi-layers. The external deposited layeris with five times longer growing period. The coatings are deposited at the temperature of $400^{\circ} \mathrm{C}$ and the work pressure of 2 Pa. Polished steel discs $(\varnothing 20 \mathrm{~mm} \times 5 \mathrm{~mm}$ thick) are used as the substrate material. The measured dry friction coeficients are in the range of $0.216-0.356$ and the coefficients friction using process fluid is in the range of $0.085-$ 0.151. The calculated wear of the counter-body is in the range of $(0.66 \div 4.21) \times 10^{-3} \mathrm{~mm}^{3}$, asit depends on the friction conditions and the used multilayer coating.

Keywords: cathodic arc evaporation, multilayer, wear rate, process fliud, friction coeficient

\section{Acknowledgement}

The paper was supported in part by the project LO1201 through the financial support of the Ministry of Education, Youth and Sports in the framework of the targeted support of the "National Programme for Sustainability I" and the OPR\&DI project “Centre for Nanomaterials, Advanced Technologies and Innovation” registration number CZ.1.05/2.1.00/01.0005.

\section{References}

[1] SHAJI, S., RADHAKRISHNAN, V. (2003). Mach. Sci. Technol., Vol. 7, pp. 137 - 155.

[2] HOLMBERG, K., MATTHEWS, A. (2009). Coatings tribology: properties, mechanisms, techniques and applications in surface engineering. Elsevier Science, xv, 560 p., [3] p. of plates. Tribology and interface engineering series, 56. ISBN 9780444527509. 2nd ed. Boston.

[3] BURAKOWSKI, T., WIERZCHON, T. (1999). Surface Engineering of Metals: Principles, Equipment, Technologies, Materials Science and Technology, p. 592.CRC Press, Boca Raton, Fla.

[4] RUGGIERO, A., VALÁŠEK, P., MEROLA, M. (2015) Friction and Wear Behaviors of Al/Epoxy Composites during Reciprocating Sliding Tests. In: Manufacturing Technology, Vol. 15, No. 4, pp. 684 - 689, ISSN 12132489.

[5] SHUM, P., TAM, W., LI. K., ZHOU, Z., SHEN, Y. (2004). Wear, Vol. 257, pp. 1030 - 1041.

[6] MUNZ, W. D., VAC. J. (1986). J. Vac. Sci. Technol. A, Vol. 4, pp. $2717-2725$.

[7] KNOTEK, O., MUNZ, W., LEYENDECKER, T., VAC. J. (1987). J. Vac. Sci. Technol. A, Vol. 5 , pp. 2173 2179.

[8] PALDEY, S., DEEVI, S.C., (2003). Mater. Sci. Eng. A, Vol. 342, pp. 58 - 79.

[9] BARSHILIA, H., DEEPTHI, B., RAJAN, K. (2006). Vacuum, Vol. 81, pp. 479 - 488

[10] BUDZYNSKI, P., SIELANKO, J., SUROWIEC, Z. (2008). Intermetallics, Vol. 16, pp. 987 - 994.

[11] JIANG, N., SHEN, Y., ZHANG, H., BAO, S., HOU, X. (2006). Materials Science and Engineering B, Vol. 135, pp. $1-9$.

[12] CHEN, L., DU, Y., WANG, SHE. Q., WANG, AI. J., XU, H. (2009). Materials Science and Engineering A, Vol. 502, pp. $139-143$.

[13] SINGH, K., LIMAYE, P., SONI, N., GROVER, A., AGRAWAL, R., SURI, A. (2005). Waer, Vol. 258, pp. 1813 $-1824$.

[14] MARQUES, L., CARVAlHO, S., VAZ, F., RAMOS, M. M. D., REBOUTA, L. (2009). Vacuum, Vol. 83, pp. $1240-1243$. 
[15] LI, Y. S., SHIMADA, S., KIYONO, H., HIROSE, A. (2006).ActaMaterialia, Vol. 54, pp. 2041 - 2048.

[16] MEI, F., SHAO, N., WEI, L., LI, G. (2005). Materials Letters, Vol. 59, pp. 2210 - 2213.

[17] CHEN, L., DU, Y., WANG, S.Q., LI, J. (2007). Int. Journal of Refractory Metals \& Hard Materials, Vol. 25, pp. $400-404$.

[18] CARVAlHO, S., REBOUTA, L., RIBEIRO, E., VAZ, F., TAVARES, C. J., ALVES, E., BARRADAS, N. P., RIVIERE, J.P. (2009). Vacuum, Vol. 83, pp. 1206 - 1212.

[19] TUILIER, M. H., PAC, M. J., COVAREL, G., ROUSSELOT, C., KHOUCHAF, L. (2007). Surface \& Coating Technology, Vol. 201, pp. 4536 - 4541.

[20] CHEN, L., DU, Y., WANG, AI J., WANG, S.Q., ZHOU, S.Z. (2009). Int. Journal of Refractory Metals \& Hard Materials, Vol. 27, pp. $718-721$.

[21] OLIVEIRA, J. C., MANAIA, A., CAVALEIRO, A., VIEIRA, M. T. (2006). Surface \& Coating Technology, Vol. 201, pp. $4073-4077$.

[22] ZHAO, W., MEI, F., DONG, Y., LI, G. (2006). J. of Mater. Processing Tachnology, Vol. 176, pp. 179 - 182.

[23] OLIVEIRA, J. C., MANAIA, A., CAVALEIRO, A. (2008). Thin Solid Films, Vol. 516, pp. 5032 - 5028.

[24] OliveirA, J. C., MANAIA, A., DiAS, J. P., CAVAlEIRO, A., TEER, D., TAYLOR, S. (2006). Surface \& Coating Technology, Vol. 200, pp. $6583-6587$.

[25] FERNANDES, C., CARVALHO, S., REBOUTA, L., VAZ, F., DENANNOT, M. F., PACAUD, J., RIVIERE, J. P., CAVALEIRO, A. (2008). Vacuum, Vol. 82, pp. 1470 - 1474.

[26] PETKOV, N., BAHCHEDZHIEV, HR., CHOLAKOVA, T. (2015).Eur. Phys. J. Appl. Phys., Vol. $70,30801$.

[27] BAKAlOVA, T., PETKOV, N., CHOLAKOVA, T., KAVAN, Fr., BAHCHEDZHIEV, Hr. (2016). Mechanical Properties of Titanium-Aluminium base multilayer coatings: Prepared for review.

[28] BAKAlOVA, T., aj. (2015). Nanoadditives SiO2 and TiO2 in Process Fluids. In: Manufacturing technology. Vol. 15, No. 4. pp. 502 - 508. ISSN 1213-2489, 1. Edition. Usti n. L.

[29] BAKALOVA, T., LOUDA, P., VOLESKÝ, L., ANDRŠOVÁ, Z. (2014). The Use of Optical Microscopy to Evaluate the Tribological Properties. In: Manufacturing Technology, Vol. 14, No. 2, pp. 256-261. ISSN 1213-2489.

[30] LIEW, W., JIE, J., YAN, L., DAYOU, J., SIPAUT, C., MADLAN, M. (2013). Procedia Engineering, Vol. 68, pp. $512-517$.

[31] BIENK, E., REITZ, H., MIKKELSEN, N. (1995). Surface and Coating Technology, Vol. 76-77, pp. 475 - 480.

[32] RODRIGUEZ, R., GARCIA, J., MEDRANO, A., RICO, M., SANCHEZ, R., MARTINEZ, R., LABRUGERE, C., LAHAYE, M., GUETTE, A. (2002). Vacuum, Vol. 67, pp. $559-566$.

[33] LIINA, L., ADOBERG, E., AARIK, L., KULU, P., VEINTHAL, R., AAL, A. (2012). Estonian Journal of Engineering, Vol. 18 pp. $193-201$. 